Adam J. Brown, MD

Department of Rheumatologic and Immunologic

Disease, Cleveland Clinic, Cleveland, $\mathrm{OH}_{\text {; }}$

Author of Rheumatology Made Ridiculously Simple;

Host of the podcast Rheuminations

\title{
Famous and not-so-famous physical findings in infectious endocarditis: A look back
}

TF YOU'RE LOOKING FOR A DISEASE that is the most quintessentially archetypal of internal medicine, it's difficult to surpass infectious endocarditis.

Gathering a thorough history, pushing and prodding a patient's spleen, pulling down the skin under the eyes to look for petechiae, hovering your face within inches of an open palm searching for a cutaneous clue to an infectious bomb dangling on the leaflet of the mitral valve-what is more emblematic of internal

Osler was

the first

to synthesize

the known data

and case

reports on

endocarditis

in a lecture

series in 1885 medicine?

See related article, page 310

The physical examination findings of infectious endocarditis are storied and known by heart by every medical student who can rattle off Osler nodes, Janeway lesions, and splinter hemorrhages without a smart phone in sight, although they may mix up which one of those lesions is painful. These findings, though famous, are rare, and the more common findings like splenomegaly and subconjunctival petechiae are less readily listed.

The report by Goff et al in this issue is a great example of the many unusual ways infectious endocarditis can present.

To better appreciate the famous and notso-famous physical examinaton findings of infectious endocarditis, it's important to look back at the evolution of the disease.

\section{OSLER'S CONTRIBUTIONS}

The understanding of endocarditis evolved rapidly after the disease was put into the forefront of medicine by Dr. William Osler in 1885. Then came advances in microbiology like the introduction of blood cultures, allowing for more rapid and accurate diagnosis. Once the diagnosis of endocarditis became more established, clinicians began recognizing subtler clues that we apply at the bedside today.

Osler placed endocarditis on the medical map with his Gulstonian lecture series on the subject in $1885 .{ }^{1}$ Before these lectures, infective endocarditis was a known entity, usually diagnosed at autopsy, but no comprehensive information existed on its presentation and natural course. Osler was the first to synthesize the known data and case reports at that time, presenting it in a cohesive way to better understand the condition. He recognized important aspects of the disease, noting the wide range of clinical presentations, the progression from an acute febrile illness leading to rapid deterioration and death. He also noted that the illness could present over months to years before death, what would later be called subacute bacterial endocarditis.

Osler also recognized that valvular abnormalities predisposed patients to endocarditis, and that a history of rheumatic fever was common.

Osler gave credit to Ontario physician Dr. J. A. Mullin for pointing out these lesions, but in 1913, Dr. F. Parkes Weber ascribed the findings to Osler. ${ }^{2}$ 


\section{JANEWAY'S CONTRIBUTIONS}

In 1899, Dr. Edward Janeway described painless lesions on the palms and soles in patients suffering from endocarditis. His objective in describing these lesions was a viable way for clinicians to differentiate endocarditis from another "malignant process" presenting with fever and weight loss. He described the lesions as "small hemorrhages with slight nodular character in the palms of the hand and soles of the feet." He did not refer to them as Janeway lesions. That was done by Dr. Emanuel Libman, who also emphasized their painless nature.

In contrast to the painless palmar Janeway lesions, Osler nodes are painful and in the pulp of the fingers and toes, and the two lesions have forever confused medical students and clinicians alike. In a 1909 issue of the Quarterly Journal of Medicine, Osler described the ephemeral nature of the lesions: "I have known them to pass away in a few hours, but more commonly they last a day, or even longer," ${ }^{3}$ and he also noted that they are painful to touch.

A debate still rages over the etiology driving both Janeway lesions and Osler nodes all these years later, ranging from septic embolic to immune complex deposition to possibly even the same etiology that just occurs at different locations (palms vs fingers). 4,5

\section{SPLINTER HEMORRHAGES}

Splinter hemorrhages are another physical finding of infectious endocarditis on the fingers. These small, dark, straight lines often at the tips of the fingernails are a notoriously nonspecific finding, seen in clinical scenarios from trauma to sepsis but made famous because their initial description was in patients with endocarditis in the 1920s.

Dr. G. Blumer was the first to use the term splinter hemorrhages in 1926 after initially finding them on 2 patients with endocarditis. He later evaluated 48 patients with endocarditis and discovered the lesions only twice, so it was quickly recognized that they were not a very sensitive marker for endocarditis as they were found in a variety of other conditions. ${ }^{6}$ Although famous, the findings on hand and foot examination in endocarditis are rare, found in fewer than $15 \%$ of cases.?

\section{LIBMAN'S CONTRIBUTIONS}

Libman was a key figure in elucidating the more common signs and symptoms of endocarditis, as well as introducing blood culture as a diagnostic tool in the United States. ${ }^{8}$

Libman was an American physician who studied microbiology in Graz, Austria, before returning to the United States to work at Mt. Sinai in New York City, where he focused on blood cultures and work with endocarditis. With blood cultures, physicians had a new tool to help recognize endocarditis earlier, and the opportunity to recognize more clinical symptoms associated with endocarditis at an earlier stage.

Libman wrote extensively on the signs and symptoms of endocarditis, recognizing the characteristic murmur, fever, splenomegaly, anemia, and transient petechiae (commonly subconjunctival). ${ }^{9}$ He used these findings to diagnose the famous Viennese composer Gustav Mahler, who was conducting the New York Symphony in 1911 when he came down with a prolonged fever. Dr. Libman noted "a loud systolic-presystolic murmur over the precordium characteristic of chronic rheumatic mitral disease, a history of prolonged low-grade fever, A debate a palpable spleen, characteristic petechiae on the conjunctivae, and slight clubbing of the fingers." 10 Blood cultures confirmed the diagnosis and Mahler decided to cross the Atlantic and die at home in Vienna at the age of 51. ${ }^{11}$

Perhaps Libman is best known for his description of noninfectious vegetations in patients with lupus erythematosus, alongside Dr. Benjamin Sacks. ${ }^{8}$

A cynic might question the importance of diagnosing endocarditis earlier in the era where antibiotics were still decades away. But it's important to note that even as progress was being made in microbiology and the recognition of endocarditis was becoming more widespread, it was still a universally fatal condition. The despair caused by the diagnosis and the seriousness that the physical examination findings had at the time are illustrated in a journal entry of a Harvard Medical student named Alfred Reinhart in 1931: "No sooner had I removed the left arm of my coat, than there was on the ventral aspect of my left wrist a sight which I shall never forget until I die. . (a)

\section{still rages over the etiology driving Janeway lesions and Osler nodes}


There greeted my eyes about fifteen or twenty bright red, slightly raised, hemorrhagic spots about 1 millimeter in diameter...I took one glance at the pretty little collection of spots... and calmly said, 'I shall be dead within six months."”12

Alfred Reinhart had a history of rheumatic fever as a child and, being a medical student, he was painfully aware that this put him at increased risk of endocarditis. He felt his fate was sealed by recognizing the rash and its relation to endocarditis, and he was correct to the month, as he died 6 months after noticing the rash on his arm. ${ }^{12}$

\section{ENTERING THE MODERN ERA}

While the early 20th century brought about increased recognition and understanding of infective endocarditis, it was not until the early 1940s with the implementation of penicillin that there was an effective treatment. The antibiotic sulfonamide preceded penicillin, but its use in endocarditis was disappointing: eg, a review in 1943 showed only 4\% of patients having resolution of the endocarditis. ${ }^{13}$ In 1944, the first published report of the use of penicillin demonstrated a near $75 \%$ resolution of disease. ${ }^{14}$ For the first time, endocar- ditis was a potentially treatable disease. Clinicians could use their diagnostic acumen to diagnose a fatal condition, implement a therapeutic agent, and potentially save the patient.

\section{OUR CURRENT UNDERSTANDING OF ENDOCARDITIS}

Infectious endocarditis is a cornerstone of internal medicine. Its history is a fascinating story that coincides with the evolution of our understanding of microbiology, and illustrates the difficulty of making this diagnosis before advanced imaging. Numerous clinicians contributed to our understanding of the disease by recognizing a broad range of physical examination clues, and over time, clinicians became more adroit at the diagnosis of endocarditis.

Until the 1940s, endocarditis was a universally fatal diagnosis. The development of penicillin quickly changed how the disease was viewed, and the decades of work detailing the diagnostic clues paid off, as patients could be appropriately diagnosed and effectively treated.

\section{DISCLOSURES}

The author reports no relevant financial relationships which, in the context of his contributions, could be perceived as a potential conflict of interest.

\section{REFERENCES}

1. Osler $\mathbf{W}$. The Gulstonian lectures, on malignant endocarditis. $\mathrm{Br}$ Med J 1885; 1(1262):467-470. doi:10.1136/bmj.1.1262.467

2. Farrior JB, Silverman ME. A consideration of the differences between a Janeway's lesion and an Osler's node in infectious endocarditis. Chest 1976; 70(2):239-243. doi:10.1378/chest.70.2.239

3. Osler W. Chronic infectious endocarditis. QJM: An International Journal of Medicine 1909; 2(2):219-230. https://doi.org/10.1093/oxfordjournals.qjmed.a069213

4. Gunson TH, Oliver GF. Osler's nodes and Janeway lesions. Australas J Dermatol 2007; 48(4):251-255. doi:10.1111/j.1440-0960.2007.00397.x

5. Parikh SK, Lieberman A, Colbert DA, Silvers DN, Grossman ME. The identification of methicillin-resistant Staphylococcus aureus in Osler's nodes and Janeway lesions of acute bacterial endocarditis. J Am Acad Dermatol 1996; 35(5 pt 1):767-768. doi:10.1016/s0190-9622(96)90746-x

6. Young JB, Will EJ, Mulley GP. Splinter haemorrhages: facts and fiction. J R Coll Physicians Lond 1988; 22(4):240-243. pmid:3230540

7. Murdoch DR, Corey GR, Hoen B, et al. Clinical presentation, etiology, and outcome of infective endocarditis in the 21st century: the International Collaboration on Endocarditis-Prospective Cohort
Study. Arch Intern Med 2009; 169(5):463-473.

doi:10.1001/archinternmed.2008.603

8. Oppenheimer BS. In Memoriam-Emanuel Libman (1872-1946). Bull N Y Acad Med 1947; 23(2):116-117.

9. Libman E, Celler HL. The etiology of subacute infective endocarditis Am J Med Sci 1910; 4:516-527.

10. Mahler Foundation. https://mahlerfoundation.org/mahler/the-man/ health/. Accessed May 6, 2021.

11. Levy D. Gustav Mahler and Emanuel Libman: bacterial endocarditis in 1911. Br Med J (Clin Res Ed) 1986; 293(6562):1628-1631. doi:10.1136/bmj.293.6562.1628

12. Flegel KM. Our medical past. Subacute bacterial endocarditis observed: the illness of Alfred S. Reinhart. CMAJ 2002; 167(12):1379 1383. pmid: 12473638

13. Lichtman SS. Treatment of subacute bacterial endocarditis: current results. Ann Intern Med 1943; 19:787.

14. Morgan WL, Bland EF. Bacterial endocarditis in the antibiotic era. Circulation 1959; 19(5):753-765. doi:10.1161/01.cir.19.5.753

Address: Adam J. Brown, MD, Department of Rheumatologic and Immunologic Disease, A50, Cleveland Clinic, 9500 Euclid Avenue, Cleveland, $\mathrm{OH}$ 44195; browna22@ccf.org 